\title{
Phasor wheels for quantitative learning of quantum physics ${ }^{\dagger}$
}

\author{
Rahul Choudhary *, David Blair
}

Publisher's Note: MDPI stays neutral with regard to jurisdictional claims in published maps and institutional affiliations.

\section{cc) (i)}

Copyright: (c) 2021 by the authors. Submitted for possible open access publication under the terms and conditions of the Creative Commons Attribution (CC BY) license (http://creativecommons.org/licenses /by/4.0/).

\begin{abstract}
There is wide recognition of the immense applications of the concepts of quantum physics in modern technologies such as solar panels, mobile phones, global positioning systems, and medical physics which is promoting the need for the introduction of these concepts at an early age. To make the concepts of quantum physics accessible to students of middle school and above, the Einstein-First project has developed tangible and tactile tools in the form of models and analogies. Until now in this project, we presented the concepts of quantum interference as a modern observational fact. To give a more comprehensive understanding of these concepts, we present a graphical approach for exploring basic quantum mechanical phenomena such as matter-wave interference, diffraction, and reflection based on Feynman's method of summation of all possible paths (path integrals). We developed tactile tools called phasor wheels which can be used to perform vector summation and obtain the probability amplitude of photons. Supported by videos of single-photon interference and matter-wave interference (such as phthalocyanine molecules), students obtain insights into the quantum world in which observations represent the quantum probability amplitudes. Results from trial programs on high school students (14-15 years old) showed the possibility of teaching these concepts.
\end{abstract}

Keywords: phasors, quantum physics, quantum weirdness, modern physics 